\title{
Full-Duplex Spectrum Sharing in Cooperative Single Carrier Systems
}

\author{
Yansha Deng*, Kyeong Jin Kim ${ }^{\dagger}$, Trung Q. Duong ${ }^{\ddagger}$, Maged Elkashlan*, \\ George K. Karagiannidis ${ }^{\S}$, and Arumugam Nallanathan $₫$ \\ *Queen Mary University of London, London, UK \\ ${ }^{\dagger}$ Mitsubishi Electric Research Laboratories (MERL), Cambridge, MA, USA \\ ${ }^{\ddagger}$ Queen’s University Belfast, Belfast, UK \\ $\S$ Khalifa University, Abu Dhabi, UAE and Aristotle University of Thessaloniki, Thessaloniki, Greece \\ `King's College London, London, UK
}

\begin{abstract}
In this paper, we propose cyclic prefix single carrier (CP-SC) full-duplex transmission in cooperative spectrum sharing to achieve multipath diversity gain and full-duplex spectral efficiency. Integrating full-duplex transmission into cooperative spectrum sharing systems results in two intrinsic problems: 1) the peak interference power constraint at the PUs are concurrently inflicted on the transmit power at the secondary source (SS) and the secondary relays (SRs); and 2) the residual loop interference occurs between the transmit and the receive antennas at the secondary relays. Thus, examining the effects of residual loop interference under peak interference power constraint at the primary users and maximum transmit power constraints at the SS and the SRs is a particularly challenging problem in frequency selective fading channels. To do so, we derive and quantitatively evaluate the exact and the asymptotic outage probability for several relay selection policies in frequency selective fading channels. Our results manifest that a zero diversity gain is obtained with full-duplex.
\end{abstract}

\section{INTRODUCTION}

Cognitive relaying [1] has emerged as a revolutionary approach to ease the spectrum utilization inefficiency, while ensure the reliable communication and coverage extension of the secondary network at the same time. Recent research and development of full-duplex relaying (FDR) without utilizing residual loop interference mitigation has attracted increasing interests, considering that FDR offers high spectral efficiency compared to HDR by transmitting and receiving signals simultaneously using the same channel [2].

Single carrier (SC) transmission [3] is currently under consideration for IEEE 802.11ad [4] and 3GPP Long-Term Evolution [5]. Owing to the fact that SC can provide a lower peak-to-average power ratio (PAPR) and power amplifier backoff [6] compared to orthogonal division multiplexing (OFDM). In addition, by adding the cyclic prefix (CP) to the front of the transmission symbol block, the multipath diversity gain can be obtained [7]. Therefore, several works have proposed the cyclic prefix single carrier (CP-SC) transmission in cooperative relaying systems [8] and cooperative spectrum sharing networks $[9,10]$.

In this paper, we introduce full-duplex relaying and relay selection in SC spectrum sharing systems to obtain the spatial diversity and the spectral efficiency. We propose three relay selection policies, namely, max-min relay selection (MM), partial relay selection (PS), and loop interference relay selection (LI). Unlike cognitive half-duplex relay network (CogHRN), in cognitive full-duplex relay network (CogFRN), the concurrent reception and transmission entails two intrinsic problems: 1) the peak interference power constraint at the PUs are concurrently inflicted on the transmit power at the SS and the SRs; and 2) the residual loop interference due to signal leakage is introduced between the transmit and the receive antennas at each SR. To examine the effects of these two problems under frequency selective fading channels, we derive the exact expressions for the outage probabilities and the asymptotic outage probabilities for three relay selection policies. In the high SNR regime, we establish that outage probability floors exist with zero diversity gains regardless of the spatial diversity offered by the relay selection policy.

\section{System AND Channel Model}

We consider a cooperative spectrum sharing network consisting of $L \mathrm{PU}$-receivers $\left(\mathrm{PU}_{1}, \ldots, \mathrm{PU}_{L}\right)$, a single $\mathrm{SS}$, a single SD, and a cluster of $K$ SRs $\left(\mathrm{R}_{1}, \ldots, \mathrm{R}_{K}\right)$. The CP-SC transmission is used in this network. Among the $K$ SRs, the best SR which fulfills the relay selection criterion is selected to forward the transmission to the SD using the amplify-andforward (AF) relaying protocol. In this network, we make the following assumptions for the channel models.

Assumption 1: We assume perfect channel state information (CSI) for all links. For the secondary channel, the instantaneous sets of channel impulse responses (CIRs) from the SS to the $k$ th $\mathrm{SR}$ and from the $k$ th $\mathrm{SR}$ to the SD composing of $N_{1, k}$ and $N_{2, k}$ multipath channels, are denoted as $\mathbf{g}_{N_{1, k}}^{s, k}=\left[g_{0}^{s, k}, \ldots, g_{N_{1, k}-1}^{s, k}\right]^{T} \in \mathbb{C}^{N_{1, k} \times 1}$ and $\mathbf{g}_{N_{2, k}}^{k, d}=$ $\left[g_{0}^{k, d}, \ldots, g_{N_{2, k}-1}^{k, d}\right]^{T} \in \mathbb{C}^{N_{2, k} \times 1}$, respectively. The instantaneous sets of CIRs from the SS to the lth PU $\left(\mathrm{PU}_{l}\right)$ and from the $k$ th SR to the $l$ th $\mathrm{PU}_{l}$ composing of $N_{3, l}$ and $N_{4, k, l}$ multipath channels, are denoted as $\mathbf{f}_{N_{3, l}}^{s, l}=\left[f_{0}^{s, l}, \ldots, f_{N_{3, l}-1}^{s, l}\right]^{T} \in$ $\mathbb{C}^{N_{3, l} \times 1}$ and $\mathbf{f}_{N_{4, k, l}}^{k, l}=\left[f_{0}^{k, l}, \ldots, f_{N_{4, k, l}-1}^{k, l}\right]^{T} \in \mathbb{C}^{N_{4, k, l} \times 1}$, respectively. All channels are composed of independent and identically distributed (i.i.d.) complex Gaussian RVs with zero means and unit variances. The maximum channel length $N_{\max }$ 
$\triangleq \max \left\{N_{1, k}, N_{2, k}, N_{3, l}, N_{4, k, l}\right\}$ is assumed to be shorter than the CP length, denoted by $N_{\mathrm{CP}}$, to restrain the interblock symbol interference (IBSI) and intersymbol interference (ISI) in single carrier transmission [6]. Accordingly, the path loss components from the SS to the $k$ th $\mathrm{SR}$, from the $k$ th $\mathrm{SR}$ to the SD, from the SS to the $\mathrm{PU}_{l}$, and from the $k$ th $\mathrm{SR}$ to the $\mathrm{PU}_{l}$ are defined as $\alpha_{1, k}, \alpha_{2, k}, \alpha_{3, l}$, and $\alpha_{4, k, l}$, respectively.

Assumption 2: For underlay spectrum sharing, the peak interference power constraint at the $l$ th $\mathrm{PU}$ is denoted as $I_{t h}$. Also, the transmit power at the SS and the SRs are restricted by the maximum transmit power constraints $P_{T}$ and $P_{R}$, respectively.

In the full-duplex mode, the PUs suffer interference from the SS and the SRs concurrently. Similar as [11], we simply assume that the maximum interference inflicted on the PUs by the SS or the SRs are set to be a half of the total peak interference power constraint at the PUs $\left(\frac{1}{2} I_{t h}=Q\right)$, where $Q$ is the maximum interference caused by the SS or the $k$ th $\mathrm{SR}$. Therefore, the transmit power at the SS and the $k$ th SR are given by

$$
\begin{aligned}
& P_{S}=\min \left(\frac{Q}{\max _{l=1, \ldots, L}\left\{\alpha_{3, l}\left\|\mathbf{f}_{N_{3, l}}^{s, l}\right\|^{2}\right\}}, P_{T}\right), \text { and } \\
& P_{R, k}=\min \left(\frac{Q}{\max _{l=1, \cdots, L}\left\{\alpha_{4, k, l}\left\|\mathbf{f}_{N_{4, k, l}}^{k, l}\right\|^{2}\right\}}, P_{R}\right) .
\end{aligned}
$$

Let $\mathbf{x}_{s} \in \mathbb{C}^{N_{s} \times 1}$ denote the transmit block symbol after applying digital modulation. We assume that $E\left\{\mathbf{x}_{s}\right\}=\mathbf{0}$ and $E\left\{\mathbf{x}_{s} \mathbf{x}_{s}^{H}\right\}=\mathbf{I}_{N_{s}}$. After appending the CP with $N_{\mathrm{CP}}$ symbols at the beginning of $\mathbf{x}_{s}$, the augmented transmit block symbol is transmitted over the frequency selective channels $\left\{\mathbf{g}_{N_{1, k}}^{s, k}\right\}$. After the removal of the CP-related received signal part, the received signal at the $k$ th $\mathrm{SR}$ is given by

$$
\mathbf{y}_{r, k}=\sqrt{P_{S} \alpha_{1, k}} \mathbf{G}_{N_{1, k}}^{s, k} \mathbf{x}_{s}+\sqrt{P_{R, k}} \mathbf{H}_{k} \mathbf{x}_{r, k}+\mathbf{n}_{s, k},
$$

where $\mathbf{G}_{N_{1, k}}^{s, k}$ is the right circulant matrix determined by the channel vector $\left[\left(\mathbf{g}_{N_{1, k}}^{s, k}\right)^{T}, \mathbf{0}_{1 \times\left(N_{s}-N_{1, k}\right)}\right]^{T} \in \mathbb{C}^{N_{s} \times 1}$. The residual loop interference channel is denoted as $\mathbf{H}_{k} \triangleq \operatorname{Diag}\left\{h_{k, 1}, \cdots, h_{k, N_{s}}\right\}$, which is a diagonal channel matrix between the transmit and receive antennas at the $k$ th $\mathrm{SR}$. It is assumed that the thermal noise received at the $k$ th relay is modeled as a complex Gaussian random variable with zero mean and variance $\sigma_{n}^{2}$, i.e., $\mathbf{n}_{s, k} \sim \mathcal{C N}\left(\mathbf{0}, \sigma_{n}^{2} \mathbf{I}_{N_{s}}\right)$.

After AF relaying, the received signal at the SD via the $k$ th $\mathrm{SR}$ is given by

$$
\mathbf{y}_{r, d}=\sqrt{\alpha_{2, k}} \mathbf{G}_{N_{2, k}}^{k, d} \mathbf{G}_{k} \mathbf{x}_{r, k}+\mathbf{n}_{r, d},
$$

where $\mathbf{G}_{N_{2, k}}^{k, d}$ is the right circulant matrix formed by $\left[\left(\mathbf{g}_{N_{2, k}}^{k, d}\right)^{T}, \mathbf{0}_{\mathbf{1} \times\left(\mathbf{N}_{\mathbf{s}}-\mathbf{N}_{\mathbf{2}, \mathbf{k}}\right)}\right]^{T} \in \mathbb{C}^{N_{s} \times 1}, \mathbf{G}_{k} \triangleq g_{k} \mathbf{I}_{N_{s}}$ is the relay gain matrix for the $k$ th $\mathrm{SR}$, and $\mathbf{n}_{r, d} \sim \mathcal{C N}\left(\mathbf{0}, \sigma_{n}^{2} \mathbf{I}_{N_{s}}\right)$. According to [8], the relay gain $g_{k}$ is given by

$$
g_{k} \triangleq \sqrt{\frac{P_{R, k}}{P_{s} \alpha_{1, k}\left\|\mathbf{g}_{N_{1, k}}^{s, k}\right\|^{2}+P_{R, k}\left|h_{k}\right|^{2}+\sigma_{n}^{2}}},
$$

where $h_{k}=\left\{h_{k, n}\right\}_{n=1}^{N_{s}}$.

Inserting (3) and (5) into (4), the e2e-SINR at the SD is upbounded as

$$
\gamma_{e 2 e}^{k}=\frac{\frac{\gamma^{s, k}}{\gamma^{k, I}+1} \gamma^{k, d}}{\frac{\gamma^{s, k}}{\gamma^{k, I}+1}+\gamma^{k, d}+1} \leq \min \left(\frac{\gamma^{s, k}}{\gamma^{k, I}+1}, \gamma^{k, d}\right),
$$

where we define the e-SNR from the SS to the $k$ th SR as $\gamma^{s, k} \triangleq \alpha_{1, k} \gamma_{s}\left\|\mathbf{g}_{N_{1, k}}^{s, k}\right\|^{2}$, the e-SNR from the $k$ th SR to the SD as $\gamma^{k, d} \triangleq \alpha_{2, k} \gamma_{k}\left\|\mathbf{g}_{N_{2, k}}^{k, d}\right\|^{2}$, and the e-INR at the $k$ th SR as $\gamma^{k, I} \triangleq \gamma_{k}\left|h_{k}\right|^{2}$. Note that $\gamma_{s} \triangleq \frac{P_{S}}{\sigma_{n}^{2}}$ and $\gamma_{k} \triangleq \frac{P_{R, k}}{\sigma_{n}^{2}}$.

\section{Distributions OF E-SNRS AND E-SINR}

In the sequel, a RV $X$ distributed according to a gamma distribution with shape $N$ and scale $\alpha$ is denoted by $X \sim \operatorname{Ga}(N, \alpha)$. Note that the magnitudes of the four channel vectors $\left\|\mathbf{g}_{N_{1, k}}^{s, k}\right\|^{2},\left\|\mathbf{g}_{N_{2, k}}^{k, d}\right\|^{2},\left\|\mathbf{f}_{N_{3, l}}^{s, l}\right\|^{2}$, and $\left\|\mathbf{f}_{N_{4, k, l}}^{k, l}\right\|^{2}$ are distributed as gamma distributions with shapes $N_{1, k}, N_{2, k}$, $N_{3, l}$, and $N_{4, k, l}$, respectively, and scale 1 . Also, $\left|h_{k}\right|^{2}$ is distributed as a gamma distribution with shape 1 and scale 1. We also define $X_{k} \triangleq \alpha_{1, k}\left\|\mathbf{g}_{N_{1}}^{s, k}\right\|^{2} \sim \mathrm{Ga}\left(N_{1, k}, \alpha_{1, k}\right)$ and $Y_{1} \triangleq \max _{l=1, \cdots, L}\left\{\alpha_{3, l}\left\|\mathbf{f}_{N_{3}}^{s, l}\right\|^{2}\right\}, \quad Y_{k} \triangleq \max _{l=1, \cdots, L}\left\{\alpha_{4, k, l}\left\|\mathbf{f}_{N_{4, k, l}}^{k, l}\right\|^{2}\right\}$, $R_{k}=\left|h_{k}\right|^{2}$.

Definition 1: Let $X_{i} \sim \operatorname{Ga}\left(N_{i}, 1\right)$, then the CDF and the PDF of a $\mathrm{RV} X_{\max } \triangleq \max \left\{a_{1} X_{1}, a_{2} X_{2}, \ldots, a_{L} X_{L}\right\}$ are given, respectively, as

$$
\begin{aligned}
& F_{X_{\max }}(x)=1+\widetilde{\sum_{L, j_{t},\left\{N_{i}\right\},\left\{a_{i}\right\}}\left[x^{\tilde{j}} e^{-b x} \mathrm{U}(x)\right] \text { and }} \\
& f_{X_{\max }}(x)=\sum_{L, j_{t},\left\{N_{i}\right\},\left\{a_{i}\right\}} e^{-b x}\left[\tilde{j} x^{\tilde{j}-1} \mathrm{U}(x)-b x^{\tilde{j}} \mathrm{U}(x)\right],
\end{aligned}
$$

where

$$
\begin{aligned}
\sum_{L, j_{t},\left\{N_{i}\right\},\left\{a_{i}\right\}}[\cdot] \triangleq & \sum_{l=1}^{L} \frac{(-1)^{l}}{l !} \underbrace{\sum_{n_{1}=1}^{L} \cdots \sum_{n_{l}=1}^{L}}_{\left|n_{1} \cup n_{2} \cup \cdots \cup n_{l}\right|=l} \sum_{j_{1}=0}^{N_{n_{1}}-1} \cdots \sum_{j_{l}=0}^{N_{n_{l}}-1} \\
& \prod_{t=1}^{l}\left(\frac{1}{j_{t} !\left(a_{n_{t}}\right)^{j_{t}}}\right)[\cdot], \tilde{j} \triangleq \sum_{t=1}^{l} j_{t}
\end{aligned}
$$

and $b \triangleq \sum_{t=1}^{l} \frac{1}{a_{n_{t}}}$, with $\left|n_{1} \cup n_{2} \cup \ldots \cup n_{l}\right|$ denoting the dimension of the union of $l$ indices $\left\{n_{1}, \ldots, n_{l}\right\}$. In the sequel, we have defined the normalized powers $\bar{\gamma}_{Q} \triangleq Q \bar{\gamma}, \bar{\gamma}_{T} \triangleq P_{T} \bar{\gamma}, \mu_{R} \triangleq \frac{Q}{P_{R}}$, and $\bar{\gamma}_{R} \triangleq P_{R} \bar{\gamma}$, with $\bar{\gamma} \triangleq \frac{1}{\sigma_{n}^{2}}$.

\section{A. e-SNR from the SS to the kth SR}

From the definition of $\gamma^{s, k} \triangleq \min \left(Q / Y_{1}, P_{T}\right) X_{k} \bar{\gamma}$, we derive the following CDF of $\gamma^{s, k}$ as

$$
\begin{aligned}
& \mathbb{F}_{\gamma^{s, k}}(\gamma)= \\
& 1-e^{-\frac{\gamma}{\alpha_{1, k} \bar{\gamma}_{T}}} \sum_{i=0}^{N_{1, k}-1} \frac{1}{i !}\left(\frac{\gamma}{\alpha_{1, k} \bar{\gamma}_{T}}\right)^{i}-\frac{\left(\gamma / \bar{\gamma}_{Q}\right)^{N_{1, k}}}{\left(\alpha_{1, k}\right)^{N_{1, k}} \Gamma\left(N_{1, k}\right)}
\end{aligned}
$$




$$
\sum_{L, j_{t},\left\{N_{3, l}\right\},\left\{\alpha_{3, l}\right\}}\left[\frac{\Gamma\left(N_{1, k}+\tilde{j}, \frac{\mu_{T} \gamma}{\alpha_{1, k} \tilde{\gamma}_{Q}}+\mu_{T} \tilde{\beta}_{1}\right)}{\left(\frac{\gamma}{\alpha_{1, k} \bar{\gamma}_{Q}}+\tilde{\beta}_{1}\right)^{N_{1, k}+\tilde{j}}}\right]
$$

where $\mu_{T} \triangleq \frac{Q}{P_{T}}, \tilde{j} \triangleq \sum_{t=1}^{l} j_{t}$, and $\tilde{\beta}_{1} \triangleq \sum_{t=1}^{l} \frac{1}{\alpha_{3, n_{t}}}$. The detailed derivation of (9) is omitted due to the page limit.

\section{B. e-SNR from the kth $S R$ to the $S D$}

Let us define $W_{k} \triangleq \alpha_{2, k}\left\|\mathbf{g}_{N_{2, k}}^{k, d}\right\|^{2} \sim \mathrm{Ga}\left(N_{2, k}, \alpha_{2, k}\right)$. Then, $\gamma^{k, d}$ is rewritten as $\gamma^{k, d} \triangleq \min \left(Q / Y_{1}, P_{R}\right) W_{k} \bar{\gamma}$ with the CDF

$$
\begin{aligned}
& \mathbb{F}_{\gamma^{k, d}}(\gamma)= \\
& 1-e^{-\frac{\gamma}{\alpha_{2, k} \bar{\gamma}_{R}}} \sum_{i=0}^{N_{2, k}-1} \frac{1}{i !}\left(\frac{\gamma}{\alpha_{2, k} \bar{\gamma}_{R}}\right)^{i}-\frac{\left(\gamma / \bar{\gamma}_{Q}\right)^{N_{2, k}}}{\left(\alpha_{2, k}\right)^{N_{2, k}} \Gamma\left(N_{2, k}\right)} \\
& \sum_{L, d_{t},\left\{N_{4, k, l}\right\},\left\{\alpha_{4, k, l}\right\}}\left[\frac{\Gamma\left(N_{2, k}+\tilde{d}, \frac{\mu_{R} \gamma}{\alpha_{2, k} \bar{\gamma}_{Q}}+\mu_{R} \tilde{\beta}_{2}\right)}{\left(\frac{\gamma}{\left(\alpha_{2, k} \bar{\gamma}_{Q}\right)}+\tilde{\beta}_{2}\right)^{N_{2, k}+\tilde{d}}}\right],
\end{aligned}
$$

where $\tilde{d} \triangleq \sum_{t=1}^{l} d_{t}$, and $\tilde{\beta}_{2} \triangleq \sum_{t=1}^{l} \frac{1}{\alpha_{4, k, n_{t}}}$.

\section{C. $e$-INR at the kth SR}

To derive the closed-form CDF of $\gamma^{k, I}$, we need to know the CDF of $Y_{k}$ based on Definition 1. Based on available CDFs of $Y_{k}$ and $R_{k}$, we can derive the CDF of $\gamma^{k, I}$ as

$$
\begin{aligned}
\mathbb{F}_{\gamma^{k, I}}(\gamma)=1-e^{-\frac{\gamma}{\bar{\gamma}_{R}}}-\frac{\gamma}{\bar{\gamma}_{Q}} & \sum_{L, d_{t},\left\{N_{4, k, l}\right\},\left\{\alpha_{4, k, l}\right\}} \frac{\Gamma\left(\tilde{d}+1, \frac{\mu_{R} \gamma}{\bar{\gamma}_{Q}}+\mu_{R} \tilde{\beta}_{2}\right)}{\left(\frac{\gamma}{\bar{\gamma}_{Q}}+\tilde{\beta}_{2}\right)^{\tilde{d}+1}} .
\end{aligned}
$$

Having derived (9) and (11) for the CDFs of the desired eSNRs, the closed-form CDF of the e-SINR $\varpi_{k} \triangleq \frac{\gamma^{s, k}}{\gamma^{k, I}+1}$ is derived in the following.

\section{Derivation of e-SINR at the kth SR}

With some manipulations, the CDF of $\varpi_{k}$ is derived as

$$
\mathbb{F}_{\varpi_{k}}(x)=\int_{0}^{\infty} \mathbb{F}_{\gamma^{s, k}}(\gamma(x+1)) \mathbb{f}_{\gamma^{k, I}}(x) d x=1-\mathcal{K}_{1}-\mathcal{K}_{2},
$$

where $\mathcal{K}_{1}$ and $\mathcal{K}_{2}$ are given in Appendix $\mathrm{A}$, due to their complex expressions. Note that now we have the CDFs of the desired e-SINR $\varpi_{k}$ and the e-SNR $\gamma^{k, d}$, which will be used to derive the e2e-SINR $\gamma_{e 2 e}^{k}$ in Section V.

\section{ASYMPTOTIC DESCRIPTION}

Throughout the asymptotic analysis, we assume $N_{1}=$ $N_{1, k}, N_{2}=N_{2, k}, N_{3}=N_{3, k}, N_{4}=N_{4, k, l}$ and $\alpha_{1}=$ $\alpha_{1, k}, \alpha_{2}=\alpha_{2, k}, \alpha_{3}=\alpha_{3, k}, \alpha_{4}=\alpha_{4, k, l}$. To examine the effect of power scaling on the outage probability, we have also defined $\rho \triangleq \frac{P_{R}}{P_{T}}$. When $\bar{\gamma}_{T} \rightarrow \infty$, we can easily observe $\bar{\gamma}_{R} \rightarrow \infty$ and $\bar{\gamma}_{Q} \rightarrow \infty$.

Notice that (6) can be simplified to one term for high SNRs. Since the second order term is dominating compared with the linear terms (i.e., $E\left[\gamma^{k, d}\right] E\left[\gamma^{k, I}\right] \gg E\left[\gamma^{k, d}\right]+E\left[\gamma^{s, k}\right]+$ $\left.E\left[\gamma^{k, I}\right]\right)$, at high SNRs, we can obtain an approximate e2eSINR expression as

$$
\gamma_{e 2 e p}^{k} \approx \frac{\gamma^{s, k} \gamma^{k, d}}{\gamma^{k, d} \gamma^{k, I}}=\frac{\gamma_{p}^{s, k}}{\gamma_{p}^{k, I}}
$$

By eliminating $\bar{\gamma}_{T}$, we derive the new expressions $\gamma_{p}^{s, k}=$ $\min \left(\frac{\mu_{T}}{Y_{1}}, 1\right) X_{k}$, and $\gamma_{p}^{k, I}=\min \left(\frac{\mu_{T}}{Y_{k}}, \rho\right) R_{k}$. We first derive the closed-form expressions for $\gamma_{p}^{s, k}$ and $\gamma_{p}^{k, I}$.

\section{A. Asymptotic e-SNR from the SS to the kth SR}

From the definition of $\gamma_{p}^{s, k}=\min \left(\frac{\mu_{T}}{Y_{1}}, 1\right) X_{k}$, we have the following asymptotic CDF of $\gamma_{p}^{s, k}$ as

$$
\begin{array}{r}
\mathbb{F}_{\gamma_{p}^{s, k}}^{\infty}(\gamma)=1-e^{-\frac{\gamma}{\alpha_{1}}} \sum_{\sum_{i=0}^{N_{1}-1} \frac{1}{i !}\left(\frac{\gamma}{\alpha_{1}}\right)^{i}-\frac{\left(\gamma / \mu_{T}\right)^{N_{1}}}{\left(\alpha_{1}\right)^{N_{1}} \Gamma\left(N_{1}\right)}} \\
\sum_{N_{t},\left\{N_{3}\right\},\left\{\alpha_{3}\right\}}\left[\frac{\Gamma\left(N_{1}+\tilde{j},\left(\frac{\gamma}{\alpha_{1} \mu_{T}}+\tilde{\beta}_{1}\right) \mu_{T}\right)}{\left(\frac{\gamma}{\alpha_{1} \mu_{T}}+\tilde{\beta}_{1}\right)^{N_{1}+\tilde{j}}}\right] .
\end{array}
$$

\section{B. Asymptotic e-INR at the kth SR}

From the definition of $\gamma_{p}^{k, I}=\min \left(\frac{\mu_{T}}{Y_{k}}, \rho\right) R_{k}$, we have the following asymptotic CDF of $\gamma_{p}^{k, I}$ as

$$
\begin{aligned}
\mathbb{F}_{\gamma_{p}^{k, I}}^{\infty}(\gamma)=1-e^{-\frac{\gamma}{\rho}}-\frac{\gamma}{\mu_{T}} & \sum_{L, d_{t},\left\{N_{4}\right\},\left\{\alpha_{4}\right\}} \frac{\Gamma\left(\tilde{d}+1,\left(\frac{\gamma}{\mu_{T}}+\tilde{\beta}_{2}\right) \frac{\mu_{T}}{\rho}\right)}{\left(\gamma / \mu_{T}+\tilde{\beta}_{2}\right)} .
\end{aligned}
$$

Having (14) and (15), we can derive the asymptotic CDF of the e2e-SINR in the following.

\section{Asymptotic e2e-SINR at the kth SR}

The asymptotic CDF of $\gamma_{e 2 e p}^{k}$ is evaluated by

$$
\mathbb{F}_{\gamma_{e 2 e p}^{k}}^{\infty}(\gamma)=\int_{0}^{\infty} \mathbb{F}_{\gamma_{p}^{s, k}}(\gamma x) \mathbb{f}_{\gamma_{p}^{k, I}}(x) d x=1-\mathcal{R}_{1}-\mathcal{R}_{2},
$$

where $\mathcal{R}_{1}$ and $\mathcal{R}_{2}$ are derived in Appendx B. Note that the CDFs in (14) and (15) are independent of $\bar{\gamma}_{Q}, \bar{\gamma}_{T}$ and $\bar{\gamma}_{R}$, therefore the derived asymptotic CDF of $\gamma_{e 2 e p}^{k}$ is also irrelevant to $\bar{\gamma}_{Q}, \bar{\gamma}_{T}$, and $\bar{\gamma}_{R}$.

\section{Outage Probability of CogFRn}

In this section, we derive the expression for the exact and asymptotic outage probabilities of CogFRN with various relay selection policies.

\section{A. $\operatorname{Cog} F R N$ with $M M$}

Let $k_{\mathrm{MM}}$ be the selected relay based on the max-min criterion, which is given as

$$
k_{\mathrm{MM}}=\arg _{k=1, \ldots, K} \max \left(\min \left(\frac{\gamma^{\mathrm{s}, \mathrm{k}}}{\gamma^{k, I}+1}, \gamma^{k, d}\right)\right) .
$$


The outage probability at a given threshold $\eta$ is given by

$$
\mathbb{P}_{\mathrm{MM}}^{\text {out }}(\eta)=\prod_{k=1}^{K}\left(1-\left(1-\mathbb{F}_{\varpi_{k}}(\eta)\right)\left(1-\mathbb{F}_{\gamma^{k, d}}(\eta)\right)\right),(18)
$$

where $\mathbb{F}_{\varpi_{k}}(x)$ and $\mathbb{F}_{\gamma^{k, d}}(x)$ are given in (12) and (10), respectively.

Based on (13), the asymptotic outage probability can be written as

$$
\mathbb{P}_{\mathrm{MM}}^{\infty, \text { out }}(\eta)=\left(\mathbb{F}_{\gamma_{e 2 e p}^{k}}^{\infty}(\eta)\right)^{K} .
$$

Substituting the derived expression of $\mathbb{F}_{\gamma_{e 2 e p}^{k}}^{\infty}(\gamma)$ in (16) at a given $\eta$ into (19), we obtain the asymptotic outage probability. Since $\mathbb{P}_{\mathrm{MM}}^{\infty, \text { out }}(\eta)$ is independent of $\bar{\gamma}_{T}, \bar{\gamma}_{R}$, and $\bar{\gamma}_{Q}$, the outage diversity gain is zero.

\section{B. $\operatorname{Cog} F R N$ with PS}

In this policy, the relay which has the maximum ratio of the e-SNR of the first hop to its e-INR is selected. Thus, the index of the selected relay is denoted as

$$
k_{\mathrm{PS}}=\arg _{k=1, \ldots, K} \max \left(\frac{\gamma^{s, k}}{\gamma^{k, I}+1}\right) .
$$

Recall that we have the same distribution for each SR to the SD link. Therefore, the outage probability is evaluated by

$$
\mathbb{P}_{\mathrm{PS}}(\eta)=1-\left(1-\mathbb{F}_{\varpi_{k}}(\eta)^{K}\right)\left(1-\mathbb{F}_{\gamma^{k_{\mathrm{PS}}, d}}(\eta)\right) .
$$

Using (10) and (12), the closed-form expression for (21) can be obtained. Moreover, the asymptotic outage probability is given by

$$
\mathbb{P}_{\mathrm{PS}}^{\infty, \text { out }}(\eta)=\left(\mathbb{F}_{\gamma_{e 2 e p}^{k}}^{\infty}(\eta)\right)^{K} .
$$

Substituting the closed-form expression of $\mathbb{F}_{\gamma_{e 2 e p}^{k}}^{\infty}(\gamma)$ in (16) at a given $\eta$ into (22), we obtain the corresponding asymptotic outage probability. As for the proportional interference constraint, the asymptotic outage probability of CogFRN with the PS policy is the same as that of the MM policy. Thus, the asymptotic diversity gain is zero.

\section{CogFRN with LI}

In the LI policy, a relay with the minimum loop interference is selected, thus the index of the selected relay is given as

$$
k_{\mathrm{LI}}=\arg _{k=1, \ldots, K} \min \left(\gamma^{k, I}\right) .
$$

The CDF of $\gamma^{k_{\mathrm{LI}}, I}$ is derived by using

$$
\mathbb{F}_{\gamma^{k} \mathrm{LI}, I}(x)=1-\left(1-\mathbb{F}_{\gamma^{k, I}}(x)\right)^{K} .
$$

Accordingly, the CDF of $\varpi_{k_{\mathrm{LI}}}=\frac{\gamma^{s, k}}{\gamma^{k \mathrm{LI}}, I+1}$ becomes

$$
\mathbb{F}_{\varpi_{k_{\mathrm{LI}}}}(\gamma)=1+\gamma \int_{0}^{\infty} \tilde{\mathbb{f}}_{\gamma^{s, k}}(\gamma(x+1)) \mathbb{F}_{\gamma^{k} \mathrm{LI}, \mathrm{I}}(x) d x,
$$

where $\mathbb{F}_{\gamma^{s, k}}(x)=1-\tilde{\mathbb{F}}_{\gamma^{s, k}}(x)$, and $\tilde{\mathbb{f}}_{\gamma^{s, k}}(x)$ is derived by taking the derivative of $\tilde{\mathbb{F}}_{\gamma^{s, k}}(x)$. Therefore, by substituting (25) and (10) into (26), the outage probability can be derived.
Unfortunately, (26) does not have a closed-form expression, so that we need to numerically evaluate it.

$$
\mathbb{P}_{L I}\left(\eta_{F}\right)=1-\left(1-\mathbb{F}_{\varpi_{k_{L I}}}(\eta)\right)\left(1-\mathbb{F}_{\gamma^{k_{\mathrm{LI}}, d}}(\eta)\right) .
$$

In the high SNR regime, the e2e-SINR expression of CogFRN with the LI policy becomes

$$
\gamma_{e 2 e p}^{k_{\mathrm{LI}}} \approx \frac{\gamma_{p}^{s, k_{\mathrm{LI}}}}{\gamma_{p}^{k_{\mathrm{LI}}, I}}=\frac{\gamma_{p}^{s, k_{\mathrm{LI}}}}{\min _{k_{\mathrm{LI}}}\left\{\gamma_{p}^{k, I}\right\}},
$$

where $\gamma_{p}^{s, k_{\mathrm{LI}}}=\min \left(\frac{\mu_{T}}{Y_{1}}, 1\right) X_{k}$, and $\gamma_{p}^{k, I}=\min \left(\frac{\mu_{T}}{Y_{k}}, \rho\right) R_{k}$. The CDF of $\gamma_{e 2 e p}^{k_{\mathrm{LI}}}=\frac{\gamma_{p}^{s, k \mathrm{LI}}}{\min _{k}\left\{\gamma_{p}^{k, I}\right\}}$ becomes

$$
\mathbb{F}_{\gamma_{e 2 e p}^{k_{\mathrm{LI}}}}^{\infty}(\gamma)=1+\gamma \int_{0}^{\infty} \tilde{\mathbb{f}}_{\gamma_{p}^{s, k}, k_{\mathrm{LI}}}(\gamma x) \mathbb{F}_{\gamma_{p}^{k_{\mathrm{LI}}, I}}(x) d x,
$$

where $\mathbb{F}_{\gamma_{p}^{s, k \mathrm{LI}}}(\gamma)=1-\tilde{\mathbb{F}}_{\gamma_{p}^{s, k \mathrm{LI}}}(x)$, and $\tilde{\mathbb{f}}_{\gamma_{p}^{s, k_{\mathrm{LI}}}}(x)$ is derived by taking the derivative of $\mathbb{\gamma}_{\gamma_{p}}^{s, k_{\mathrm{LI}}}(x)$.

Thus, the asymptotic outage probability can be derived by substituting the derived expression of $\tilde{\mathbb{H}}_{\gamma_{p}^{s, k_{\mathrm{LI}}}}(x)$ and (15) into $\mathbb{P}_{\mathrm{LI}}^{\infty}(\eta)=\mathbb{F}_{\gamma_{e 2 e p}^{k_{\mathrm{LI}}}}^{\infty}(\eta)$. A closed-form solution is not tractable. However, we see that the expressions of $\mathbb{F}_{\gamma_{v}^{s, k}}^{\infty}(\gamma)$

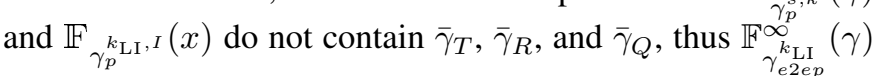
is constant over all SNR values. Therefore, we conclude that in CogFRN, the outage diversity gain of the LI policy is identical to those of the MM and the PS policies.

\section{Vi. Simulation Results}

In this section, we present numerical results to verify our new analytical results for the proposed relay selection policies in cooperative CP-SC spectrum sharing systems. We assume the symbol block size as $N_{s}=512$ and $\mathrm{CP}$ length as $N_{\mathrm{CP}}=16$. We set $\bar{\gamma}_{R}=\rho \bar{\gamma}_{T}, \bar{\gamma}_{Q}=2 \bar{\gamma}_{T}, R_{T}=1 \mathrm{bit} / \mathrm{s} / \mathrm{Hz}$, and $\eta=2^{R_{T}}-1$ in the simulations. In all the figures, we assume $\left\{N_{3}, \alpha_{3}\right\}=\{2,0.5\}$, and $\left\{N_{4}, \alpha_{4}\right\}=\{3,0.3\}$. The exact plots with MM, PS, and LI relay selection policies are numerically evaluated using (18), (21), and (26). The asymptotic outage probabilities are plotted from (19), (22), and (28).

In Fig. 1, we examine the outage probability of CogFRN with various relay selection policies and various values of $\rho$. First, we observe error floors in the high SNR region with zero outage diversiy gain. Second, for the same value of $\rho$, we find that MM outperforms PS and PS outperforms LI over all SNR values. Moreover, the MM policy converges to the same error floor as the PS policy. This can be explained by the same asymptotic outage probability derived in (19) and (22). Furthermore, we observe that as $\rho$ decreases, the outage probability with the PS policy and the LI policy decrease due to the residual loop interference.

In Fig. 2, we examine the impact of fading severity and channel size of the secondary network on the outage performance of CogFRN with the MM policy. It is easy to see that for the same fading severity, the outage probability decreases as the channel length increases but it increases as the channel 


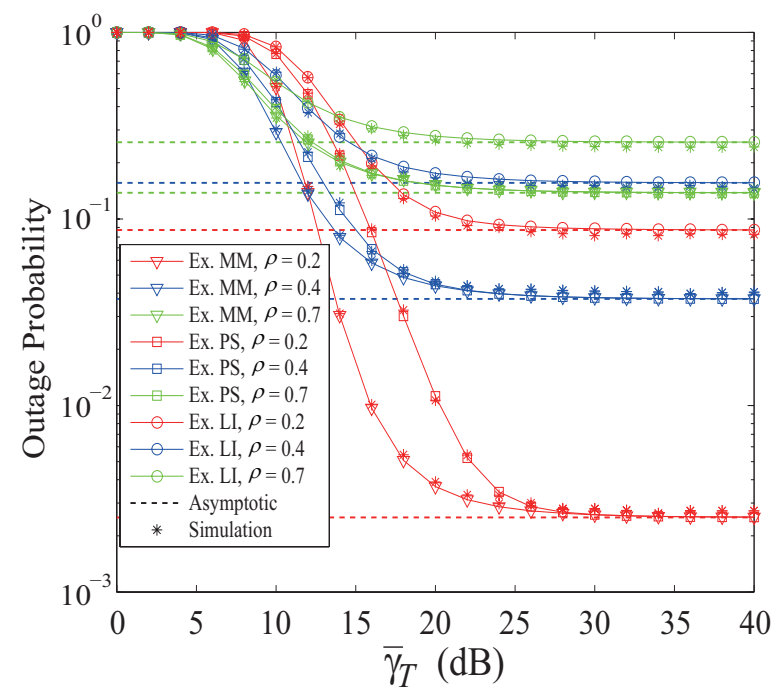

Fig. 1. Outage probability in $\operatorname{Cog} F \mathrm{RN}$ for various values of $\rho$ with $L=2$, $K=8,\left\{N_{1}, \alpha_{1}\right\}=\{2,0.1\}$, and $\left\{N_{2}, \alpha_{2}\right\}=\{3,0.1\}$.

fading parameter decreases. It is readily observed that the outage diversity gain is zero regardless of $N_{1}, N_{2}, \alpha_{1}$, and $\alpha_{2}$.

\section{CONCLUSIONS}

We have examined the effects of residual loop interference in cooperative CP-SC spectrum sharing with FDR. The outage probabilities and asymptotic outage probabilities of the MM policy requiring global CSI, as well as the PS and the LI policies requiring partial CSI have been derived and quantitatively compared. Based on these, we have confirmed that the outage diversity gain of $\operatorname{Cog} F D R$ is zero.

\section{APPENDIX A: DetaILED DERIVATION OF (12)}

The first term is evaluated as

$$
\begin{aligned}
& \mathcal{K}_{1}=e^{-\frac{\gamma}{\alpha_{1, k} \bar{\gamma}_{T}}} \sum_{i=0}^{N_{1, k}-1} \frac{1}{i !}\left(\frac{\gamma}{\alpha_{1, k} \bar{\gamma}_{T}}\right)^{i} \sum_{t=0}^{i}\left(\begin{array}{l}
i \\
t
\end{array}\right)\left[\frac{1}{\bar{\gamma}_{T}}\right. \\
& \left(\frac{1}{\bar{\gamma}_{R}}+\frac{\gamma}{\alpha_{1, k} \bar{\gamma}_{T}}\right)^{-(t+1)} \Gamma(t+1)-\frac{1}{\bar{\gamma}_{Q}} \sum_{L, d_{t},\left\{N_{4, k, l}\right\},\left\{\alpha_{4, k, l}\right\}} \\
& {\left[c_{2} \sum_{r, w} \tilde{\beta}_{2}^{-\tilde{d}-1} \Omega\left(w t+1, w t+1-\tilde{d}, \bar{\gamma}_{Q} \tilde{\beta}_{2}, \frac{1}{\bar{\gamma}_{R}}+\frac{\gamma}{\alpha_{1, k} \bar{\gamma}_{T}}\right)\right]} \\
& +\frac{1}{\bar{\gamma}_{Q}^{2}} \sum_{L, d_{t},\left\{N_{4, k, l}\right\},\left\{\alpha_{4, k, l}\right\}}\left[c_{3} \sum_{r, w} \tilde{\beta}_{2}^{-\tilde{d}-2} \Omega(w t+2, w t+1-\tilde{d},\right. \\
& \left.\left.\left.\bar{\gamma}_{Q} \tilde{\beta}_{2}, \frac{1}{\bar{\gamma}_{R}}+\frac{\gamma}{\alpha_{1, k} \bar{\gamma}_{T}}\right)\right]\right],
\end{aligned}
$$

where $\Omega(\vartheta, \tau, \zeta, v)=(\zeta)^{\vartheta} \Gamma(\vartheta) \Psi(\vartheta, \tau ; v \zeta), w t \triangleq w+t$, $c_{2} \sum_{r, w} \triangleq(\tilde{d}) ! e^{-\mu_{R} \tilde{\beta}_{2}} \sum_{r=0}^{\tilde{d}} \sum_{w=0}^{r} \frac{\left(\mu_{R}\right)^{r}}{r !}\left(\begin{array}{c}r \\ w\end{array}\right)\left(\frac{1}{\bar{\gamma}_{Q}}\right)^{w} \tilde{\beta}_{2}^{r-w}, \quad$ and

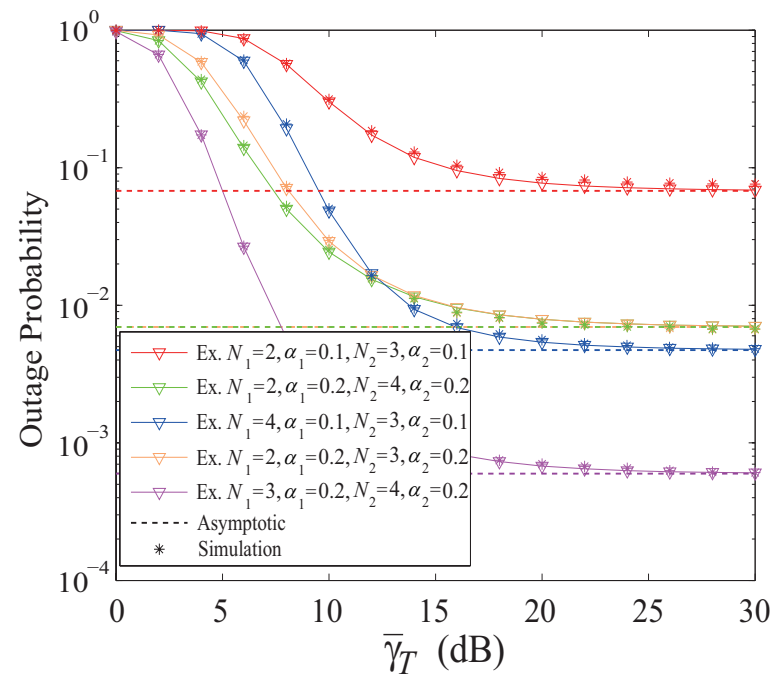

Fig. 2. Outage probability of the max-min relay selection for various channel lengths of secondary network in $\operatorname{CogFRN}: L=2, K=8$, and $\rho=0.5$.

$c_{3} \sum_{r, w} \triangleq(\tilde{d}+1) ! e^{-\mu_{R} \tilde{\beta}_{2}} \sum_{r=0}^{\tilde{d}+1} \sum_{w=0}^{r} \frac{\left(\mu_{R}\right)^{r}}{r !}\left(\begin{array}{c}r \\ w\end{array}\right)\left(\frac{1}{\bar{\gamma}_{Q}}\right)^{w} \tilde{\beta}_{2}^{r-w}$. Now the term $\mathcal{K}_{2}$ is evaluated in (A.1) at the top of the next page. In (A.1), $b_{l_{1}}=\frac{(-1)^{\tilde{d}+1-l_{1}}\left(\begin{array}{c}\tilde{d}-l_{1}+N_{1, k}+\tilde{j} \\ \tilde{j}+1-1\end{array}\right)}{\left(1+\frac{\alpha_{1, k} \bar{\gamma}_{Q} \tilde{\beta}_{1}}{\gamma}-\bar{\gamma}_{Q} \tilde{\beta}_{2}\right)^{\tilde{d}-l_{1}+N_{1, k}+\tilde{j}+1}}$, $b_{l_{2}}=\frac{(-1)^{\tilde{j}+N_{1, k}-l_{2}}\left(\begin{array}{c}\tilde{j}-l_{2}+N_{1, k}+\tilde{d} \\ \tilde{d}\end{array}\right)}{\left(-1-\frac{\alpha_{1, k} \bar{\gamma}_{Q} \tilde{\beta}_{1}}{\gamma}+\bar{\gamma}_{Q} \tilde{\beta}_{2}\right)^{\tilde{j}-l_{2}+N_{1, k}+\tilde{d}+1}}, w \tilde{h} \triangleq w+\tilde{h}, b_{l_{3}}=$ $\frac{(-1)^{\tilde{d}+2-l_{3}}\left(\begin{array}{c}\tilde{d}^{\gamma} l_{3}+N_{1, k}+\tilde{j}+1 \\ \tilde{d}+2-l_{3}\end{array}\right)}{\left(1+\frac{\alpha_{1, k} \tilde{\gamma}^{\beta_{1}}}{\gamma}-\bar{\gamma}_{Q} \tilde{\beta}_{2}\right)^{\tilde{d}-l_{3}+N_{1, k}+\tilde{j}+2}}, \quad c_{5} \triangleq \bar{\gamma}_{Q}^{\tilde{d}+1}\left(\frac{\alpha_{1, k} \bar{\gamma}_{Q}}{\gamma}\right)^{N_{1, k}+\tilde{j}}$, $b_{l_{4}}=\frac{(-1)^{\tilde{j}+N_{1, k}-l_{4}}\left(\begin{array}{c}\tilde{j}-l_{4}+N_{1, k}+\tilde{d}+1 \\ \tilde{d}+1\end{array}\right)}{\left(-1-\frac{\alpha_{1, k} \tilde{\gamma}^{\beta_{1}}}{\gamma}+\bar{\gamma}_{Q} \tilde{\beta}_{2}\right)^{\tilde{j}-l_{4}+N_{1, k}+\tilde{d}+2}}, \quad c_{6}=c_{5} \bar{\gamma}_{Q}$, and $c_{4} \sum_{m, n, \tilde{h}} \triangleq \frac{\left(N_{1, k}+\tilde{j}-1\right) !}{e^{\left(\frac{\mu_{T} \gamma}{\alpha_{1, k} \gamma}+\mu_{T} \tilde{\beta}_{1}\right)}} \sum_{m=0}^{N_{1, k}+\tilde{j}-1} \sum_{n=0}^{m} \sum_{\tilde{h}=0}^{N_{1, k}+n} \frac{\left(\mu_{T}\right)^{m}}{m !}\left(\begin{array}{c}m \\ n\end{array}\right)$ $\tilde{\beta}_{1}^{m-n}\left(\frac{\gamma}{\alpha_{1, k} \bar{\gamma}_{Q}}\right)^{n}\left(\begin{array}{c}N_{1, k}+n \\ \tilde{h}\end{array}\right)$.

\section{APPENDIX B: DETAILED DERIVATION OF (16)}

The first term $\mathcal{R}_{1}$ is evaluated as

$\mathcal{R}_{1}=$

$\sum_{i=0}^{N_{1}-1} \frac{1}{i !}\left(\frac{\gamma}{\alpha_{1}}\right)^{i}[\frac{1}{\rho}\left(\frac{1}{\rho}+\frac{\gamma}{\alpha_{1}}\right)^{-i-1} \Gamma(i+1)-\overbrace{L, d_{t},\left\{N_{4}\right\},\left\{\alpha_{4}\right\}}$

$\mu_{T}^{w i} \tilde{\beta}_{2}^{w i-\tilde{d}}\left[\sum_{r=0}^{\tilde{d}} \sum_{w=0}^{r} \Upsilon\left(\tilde{d}, \frac{\mu_{T}}{\rho}, \frac{1}{\mu_{T}}\right) \Gamma(w i+1) \Psi(w i+1\right.$,

$\left.w i+1-\tilde{d},\left(\frac{1}{\rho}+\frac{\gamma}{\alpha_{1}}\right) \mu_{T} \tilde{\beta}_{2}\right)-\sum_{r=0}^{\tilde{d}+1} \sum_{w=0}^{r} \Upsilon\left(\tilde{d}+1, \frac{\mu_{T}}{\rho}, \frac{1}{\mu_{T}}\right)$

$\left.\left.\Gamma(w i+2) \Psi\left(w i+2, w i+1-\tilde{d},\left(\frac{1}{\rho}+\frac{\gamma}{\alpha_{1}}\right) \mu_{T} \tilde{\beta}_{2}\right)\right]\right]$,

where $w i \triangleq w+i$. 


$$
\begin{aligned}
\mathcal{K}_{2}= & \frac{\left(\frac{\gamma}{\bar{\gamma}_{Q}}\right)^{N_{1, k}}}{\left(\alpha_{1, k}\right)^{N_{1, k}} \Gamma\left(N_{1, k}\right)} \sum_{L, j_{t},\left\{N_{3, l}\right\},\left\{\alpha_{3, l}\right\}}\left[c _ { 4 } \sum _ { m , n , \tilde { h } } \left[\frac { 1 } { \overline { \gamma } _ { R } } ( \frac { \gamma } { \alpha _ { 1 , k } \overline { \gamma } _ { Q } } + \tilde { \beta } _ { 1 } ) ^ { - N _ { 1 , k } - \tilde { j } } \Omega \left(\tilde{h}+1, \tilde{h}+2-N_{1, k}-\tilde{j}\right.\right.\right. \\
& \left.\left.\left(\frac{\gamma}{\alpha_{1, k} \bar{\gamma}_{Q}}+\tilde{\beta}_{1}\right) \frac{\alpha_{1, k} \bar{\gamma}_{Q}}{\gamma}, \frac{1}{\bar{\gamma}_{R}}+\frac{\gamma}{\alpha_{1, k} \bar{\gamma}_{T}}\right)\right)-\frac{1}{\bar{\gamma}_{Q}} \int_{L, d_{t},\left\{N_{4, k, l}\right\},\left\{\alpha_{4, k, l}\right\}}\left[c _ { 2 } \sum _ { r , w } c _ { 5 } \left[\sum_{l_{1}=1}^{\tilde{d}+1} b_{l_{1}}\left(\bar{\gamma}_{Q} \tilde{\beta}_{2}\right)^{-l_{1}}\right.\right. \\
& \Omega\left(w \tilde{h}+1, w \tilde{h}+2-l_{1}, \bar{\gamma}_{Q} \tilde{\beta}_{2}, \frac{1}{\bar{\gamma}_{R}}+\frac{\gamma}{\alpha_{1, k} \bar{\gamma}_{T}}\right)+\sum_{l_{2}=1}^{N_{1, k}+\tilde{j}} b_{l_{2}}\left(1+\frac{\alpha_{1, k} \bar{\gamma}_{Q} \tilde{\beta}_{1}}{\gamma}\right)^{-l_{2}} \Omega(w \tilde{h}+1, \\
& \left.\left.\left.w \tilde{h}+2-l_{2}, 1+\frac{\alpha_{1, k} \bar{\gamma}_{Q} \tilde{\beta}_{1}}{\gamma}, \frac{1}{\bar{\gamma}_{R}}+\frac{\gamma}{\alpha_{1, k} \bar{\gamma}_{T}}\right)\right]\right]+\frac{1}{\bar{\gamma}_{Q}^{2}} \sum_{L, d_{t},\left\{N_{4, k, l}\right\},\left\{\alpha_{4, k, l}\right\}}\left[c _ { 3 } \sum _ { r , w } c _ { 6 } \left[\sum_{l_{3}=1}^{\tilde{d}+2} b_{l_{3}}\left(\bar{\gamma}_{Q} \tilde{\beta}_{2}\right)^{-l_{3}}\right.\right. \\
& \Omega\left(w \tilde{h}+2, w \tilde{h}+3-l_{3}, \bar{\gamma}_{Q} \tilde{\beta}_{2}, \frac{1}{\bar{\gamma}_{R}}+\frac{\gamma}{\alpha_{1, k} \bar{\gamma}_{T}}\right)+\sum_{l_{4}=1}^{N_{1, k}+\tilde{j}} b_{l_{4}}\left(1+\frac{\alpha_{1, k} \bar{\gamma}_{Q} \tilde{\beta}_{1}}{\gamma}\right)^{-l_{4}} \Omega\left(w \tilde{h}+2, w \tilde{h}_{n}+3-l_{4},\right. \\
& \left.\left.\left.\left.\left.1+\frac{\alpha_{1, k} \bar{\gamma}_{Q} \tilde{\beta}_{1}}{\gamma}, \frac{1}{\bar{\gamma}_{R}}+\frac{\gamma}{\alpha_{1, k} \bar{\gamma}_{T}}\right)\right]\right]\right]\right]
\end{aligned}
$$

The second term $\mathcal{R}_{2}$ is evaluated via [12, Eq. 9.211.4] as

$$
\begin{aligned}
& \mathcal{R}_{2}=\overbrace{L, j_{t},\left\{N_{3}\right\},\left\{\alpha_{3}\right\}} \sum_{m=0}^{N_{1}+\tilde{j}-1} \sum_{n=0}^{m} \Phi\left(\mu_{T}\right)\left[\frac { 1 } { \rho } \tilde { \beta } _ { 1 } ^ { - N _ { 1 } - \tilde { j } } \lambda \left(N_{1}+n+1,\right.\right. \\
& \left.n+2-\tilde{j}, \frac{\alpha_{1} \mu_{T} \tilde{\beta}_{1}}{\gamma}\right)-\sum_{L, d_{t},\left\{N_{4}\right\},\left\{\alpha_{4}\right\}}\left[\frac{1}{\mu_{T}} \sum_{r=0}^{\tilde{d}} \sum_{w=0}^{r} \Upsilon\left(\tilde{d}, \frac{\mu_{T}}{\rho}, \frac{1}{\mu_{T}}\right)\right. \\
& e_{1}\left[\sum_{l_{1}=1}^{\tilde{d}+1} c_{l_{1}}\left(\mu_{T} \tilde{\beta}_{2}\right)^{-l_{1}} \lambda\left(w N_{1} n+1, w N_{1} n+2-l_{1}, \mu_{T} \tilde{\beta}_{2}\right)+\right. \\
& \left.\sum_{l_{1}+\tilde{j}} c_{l_{2}}\left(\frac{\alpha_{1} \mu_{T} \tilde{\beta}_{1}}{\gamma}\right){ }^{-l_{2}} \lambda\left(w N_{1} n+1, w N_{1} n+2-l_{2}, \frac{\alpha_{1} \mu_{T} \tilde{\beta}_{1}}{\gamma}\right)\right] \\
& -\frac{1}{\mu_{T}^{2}} \sum_{r=0} \sum_{w=0}^{r} \Upsilon\left(\tilde{d}+1, \frac{\mu_{T}}{\rho}, \frac{1}{\mu_{T}}\right) \mu_{T} e_{1}\left[\sum_{l_{3}=1}^{\tilde{d}+2} d_{l_{3}}\left(\mu_{T} \tilde{\beta}_{2}\right)^{-l_{3}}\right. \\
& \lambda\left(w N_{1} n+2, w N_{1} n+3-l_{3}, \mu_{T} \tilde{\beta}_{2}\right)+\sum_{l_{4}=1}^{N_{1}+\tilde{j}} d_{l_{4}}\left(\frac{\alpha_{1} \mu_{T} \tilde{\beta}_{1}}{\gamma}\right) \\
& \left.\left.\lambda\left(w N_{1} n+2, w N_{1} n+3-l_{4}, \frac{\alpha_{1} \mu_{T} \tilde{\beta}_{1}}{\gamma}\right)\right]\right]
\end{aligned}
$$

where $\Upsilon(\sigma, \tau, \varepsilon)=\sigma ! e^{-\tilde{\beta}_{2} \tau}\left(\begin{array}{c}r \\ w\end{array}\right) \frac{\tau^{r}}{r !} \varepsilon^{w} \tilde{\beta}_{2}^{r-w}, \Phi(\delta)=$ $\frac{(\gamma / \delta)^{N_{1}}\left(N_{1}+\tilde{j}-1\right) ! \mu^{m}}{\left(\alpha_{1}\right)^{N_{1}} \Gamma\left(N_{1}\right) m !}\left(\begin{array}{c}m \\ n\end{array}\right)\left(\frac{\gamma}{\alpha_{1, k} \delta}\right)^{n} e^{-\tilde{\beta}_{1} \delta} \tilde{\beta}_{1}^{m-n}, \quad \lambda(\vartheta, \tau, \zeta)=$ $\Omega\left(\vartheta, \tau, \zeta, \frac{1}{\rho}+\frac{\gamma}{\alpha_{1}}\right), e_{1} \triangleq \mu_{T}^{\tilde{d}+1}\left(\frac{\alpha_{1} \mu_{T}}{\gamma}\right)^{N_{1}+\tilde{j}}, w N_{1} n \triangleq w+$ $N_{1}+n$,

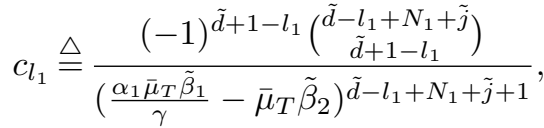

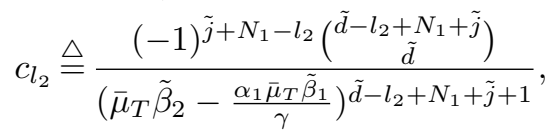

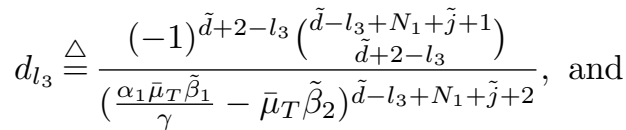

$$
\begin{aligned}
& d_{l_{4}} \triangleq \frac{(-1)^{\tilde{j}+N_{1}-l_{4}}\left(\begin{array}{c}
\tilde{d}-l_{4}+N_{1}+\tilde{j}+1 \\
\tilde{d}+1
\end{array}\right)}{\left(\bar{\mu}_{T} \tilde{\beta}_{2}-\frac{\alpha_{1} \bar{\mu}_{T} \tilde{\beta}_{1}}{\gamma}\right)^{\tilde{d}-l_{4}+N_{1}+\tilde{j}+2}} .
\end{aligned}
$$

\section{REFERENCES}

[1] J. Mitola and G. Q. Maguire, "Cognitive radio: making software radios more personal," IEEE Personal Commun. Mag., vol. 6, no. 4, pp. 13-18, Aug. 1999.

[2] I. Krikidis, H. Suraweera, P. Smith, and C. Yuen, "Full-duplex relay selection for amplify-and-forward cooperative networks," IEEE Trans. Wireless Commun., vol. 11, no. 12, pp. 4381-4393, Dec. 2012.

[3] Y.-C. Liang, A. Nallanathan, H. K. Garg et al., "Optimal training sequences for channel estimation in bi-directional relay networks with multiple antennas," IEEE Trans. Wireless Commun., vol. 58, no. 2, pp. 474-479, Feb. 2010.

[4] IEEE P802.11ad/D0.1, "Wireless lan medium access control (MAC) and physical layer (PHY) specifications: Enhancements for very high throughput in the $60 \mathrm{GHz}$ band," Jun. 2010.

[5] S. Sesia, I. Toufik, and M. Baker, LTE: The UMTS Long Term Evolution: From Theory to Practice. West Sussex, United Kingdom: John Wiley and Sons Ltd, 2009.

[6] S. Kato, H. Harada, R. Funada, T. Baykas, C. S. Sum, J. Wang, and M. A. Rahman, "Single carrier transmission for multi-gigabit $60-\mathrm{GHz}$ WPAN systems," IEEE J. Sel. Areas Commun., vol. 27, no. 8, pp. 14661478, Oct. 2009.

[7] B. Devillers, J. Louveaux, and L. Vandendorpe, "About the diversity in cyclic prefixed single-carrier systems," Elsevier Physical Commun. J., vol. 1, no. 4, pp. 266-276, Dec. 2008.

[8] K. J. Kim, T. A. Tsiftsis, and H. V. Poor, "Power allocation in cyclic prefixed single-carrier relaying systems," IEEE Trans. Wireless Commun., vol. 10, no. 7, pp. 2294-2305, Jun. 2011.

[9] K. J. Kim, T. Q. Duong, and X.-N. Tran, "Performance analysis of cognitive spectrum-sharing single-carrier systems with relay selection," IEEE Trans. Signal Process., vol. 60, no. 12, pp. 6435-6449, Dec. 2012.

[10] K. J. Kim, T. Q. Duong, H. V. Poor, and L. Shu, "Performance analysis of cyclic prefixed single-carrier spectrum sharing relay systems in primary user interference," IEEE Trans. Signal Process., vol. 60, no. 12, pp. 6729-6734, Dec. 2012.

[11] H. Kim, S. Lim, H. Wang, and D. Hong, "Optimal power allocation and outage analysis for cognitive full duplex relay systems," IEEE Trans. Wireless Commun., vol. 11, no. 10, pp. 3754-3765, Oct. 2012.

[12] I. S. Gradshteyn and I. M. Ryzhik, Table of Integrals, Series, and Products. New York: Academic Press, 2007. 